\title{
Erratum to: The Role of Social Values, Accounting Values and Institutions in Determining Accounting Conservatism
}

\author{
Stephen B. Salter • Tony Kang • Giorgio Gotti • \\ Timothy S. Doupnik
}

\section{Erratum to: Manag Int Rev}

DOI 10.1007/s11575-012-0152-1

The original version of this article unfortunately contained a mistake. The presentation of equation 3 was incomplete. The complete equation is given below.

$$
\begin{aligned}
& \operatorname{CONS}_{y, t}=\beta_{0}+\beta_{1} \text { UAI }_{y, t}+\beta_{2} \text { COLLECTIVISM }_{y, t}+\beta_{3} \text { FEMININITY }_{y, t} \\
& +\beta_{4} \text { ANTI-DIRECTOR RIGHTS } \text { R }_{y, t}+\beta_{5} \text { SELF-DEALING }{ }_{y, t} \\
& +\beta_{6} \text { iNVESTOR PROTECTIONW } B_{y, t}+\beta_{7} \text { RULELAW }_{y, t} \\
& +\beta_{8} \text { CORRUPT }_{y, t}+\beta_{9} \text { CREDITOR }_{y, t}+\beta_{10} \text { MKTCAP }_{y, t} \\
& +\beta_{11} B M_{y, t}+\beta_{12} \text { ORIGIN }_{y, t}+\epsilon
\end{aligned}
$$

Published online: 08.03.2013

(C) Springer Fachmedien Wiesbaden 2013

The online version of the original article can be found under doi:http://dx.doi.org/10.1007/s11575-012-0152-1

Prof. S. B. Salter $(\square) \cdot$ Asst. Prof. G. Gotti

College of Business, University of Texas at El Paso, El Paso, USA

e-mail: sbsalter@utep.edu

Assoc. Prof. T. Kang

School of Accounting, Oklahoma State University, Stillwater, USA

Prof. T. S. Doupnik

University of South Carolina, Columbia, USA 